\title{
Path Coefficient Analysis for Grain and Oil Yield in Coriander
}

\author{
M.K. Nagappa, N. Emmanuel, M. Lakshminarayana Reddy and A.V.D. Dorajeerao* \\ Dr. YSR University HC \& RI Venkataramannagudem, Tadepalligudem, West Godavari, \\ Andhra Pradesh-534101, India \\ *Corresponding author
}

\section{A B S T R A C T}

Keywords

Correlation, Yield

attributes,

Coriander

genotypes

Article Info

Accepted:

12 December 2017

Available Online:

10 January 2018
The present study was conducted during rabi season in the year 2015-2016 at HCRI Venkataramannagudem, Andhra Pradesh. Thirty coriander (Coriandrum sativum L.) genotypes were evaluated to estimate the correlation coefficient in Randomized Complete Block Design with two replications. grain yield per plant exhibited the path analysis indicated that leaf area, fresh weight, dry weight, number of umbels per plant, number of umbellets per umbel, umbel diameter, number of schizocarps per umbel, number of schizocarps per plant, days taken to maturity, herbage yield, harvest index, thousand seed weight and oil content had direct positive effects on grain yield per plant at phenotypic and genotypic level. Therefore, great emphasis should be given for aforesaid characters while selecting for growth and yield related traits.

\section{Introduction}

Coriander (Coriandrum sativum L.) is a native of Mediterranean region wherefrom its spread to Europe, Asia, North and South - America and Australia. It is the most important seed spice crop cultivated throughout the world both for seed and leaf purpose. It is grown in more than fifty countries with India at ranking 1st, both in area and production followed by Mexico, China, former Soviet Union, Central America and South America (Morales-Payan, 2011). The crop grows in tropics and requires a cool but comparatively dry frost-free climate, particularly at flowering and seed formation stages (Sharma and Sharma, 2004).
It is grown in almost all the states of India either for grain or leaf or dual purpose. In India the crop is cultivated mainly in Rajasthan, Madhya Pradesh, Andhra Pradesh, Orissa, Tamil Nadu and Karnataka on an area of 5.43 lakh ha with a production of 5.24 lakh metric tonnes (Tiwari, 2014). The average crop productivity is only $965 \mathrm{~kg} \mathrm{ha}^{-1}$ and is much lower in rainfed farming situation (477 $\left.\mathrm{kg} \mathrm{ha}^{-1}\right)$. The low productivity under rainfed situation is mainly due to terminal moisture stress that affects growth and productivity. Growing coriander in rainfed in Godavari zone farming situation demands highly productive types with short (75 days) to medium (85-100 days) duration for 
cultivation. Locally grown indigenous genotypes are low in productivity and give poor returns to the farmers. Critical evaluation of available selections of improved types with high yield potential/ traits is of great value to the breeder for crop improvement (Moniruzzaman, 2013). Mengesha and Getinetalemaw (2010) evaluated some Ethiopian coriander genotypes and reported that identification and evaluation of elite or promising genotypes for yield and quality is an important crop improvement strategy. Sarada and Giridhar $(2009,2011)$ opined that it is possible to realize $1500 \mathrm{~kg} \mathrm{ha}^{-1}$ under rainfed conditions if a proper combination of genotypes and management are available to the farmers. Keeping this in view, the present study was undertaken to evaluate promising diverse genotypes from Godavri zone of Andhra Pradesh. Correlation will establishes the extent of association between yield and its component and also bring out the relative importance gives a clear understanding of their association with yield. Keeping this in view, the present investigation was done to know the association among characters analysis in coriander.

\section{Materials and Methods}

The present investigation entitled "Evaluation of Coriander (Coriandrum sativum L.) Genotypes in Godavari Zone of Andhra Pradesh" was carried out during the year 2015-16 at Horticulture College and Research Institute, Dr. Y.S.R Horticultural University, Venkataramannagudem, West Godavari District. The location falls under Agroclimatic zone-10, humid, East Coast Plain and Hills (Krishna-Godavari zone) with an average annual rainfall of $900 \mathrm{~mm}$ at an altitude of $34 \mathrm{~m}$ (112 feet) above mean sea level. The geo-graphical situation is $16^{\circ} 63^{\prime}$ $120 " \mathrm{~N}$ latitude and $81^{\circ} 27^{\prime} 568^{\prime \prime}$ E longitude. It experiences hot humid summer and mild winter. A total of thirty genotypes were taken for evaluation study out of which fifteen genotypes were sourced from HRS Devihosur (Haveri) Karnataka (Ranibennur-1, Ranibennur-2, Ranibennur-3, Byadagi-1, Hangel-1, Hangel-2, Savanur-1, Savanur-2, Savanur-3, Hirekerur-1, Hirekerur-2, Hirekerur-3, Shiggaon-1, Shiggaon-2, Shiggaon-3) whereas, the rest of the accessions were sourced from HRS Lam Guntur, Andhra Pradesh (LCC-200, LCC-331, LCC-321, LCC-323, LCC-325, LCC-334, LCC-335, LCC-316, LCC-328, LCC-320, LCC-317, LCC-319 and LCC-322; and two checks viz., AD-1 (local check) and Suguna (commercial check). The experiment was laid out in RBD with two replications and thirty genotypes. The observations were recorded on various growth, seed yield and quality parameters. The crop was raised at a plant spacing of $30 \mathrm{~cm} \times 15 \mathrm{~cm}$. The seed were sown during 2nd of November and harvested during $2^{\text {nd }}$ fortnight of February. A basal fertilizer dose of $35 \mathrm{~kg} \mathrm{~N}, 35 \mathrm{~kg}$ P2O5 and 35 $\mathrm{kg} \mathrm{K}_{2} \mathrm{O} \mathrm{ha}{ }^{-1}$ was given at the time of soil preparation each year. Soil was prepared to a fine tilth and the seed sown in rows using a labor. At 20 days after sowing (DAS), the plants were thinned $15 \mathrm{~cm}$ apart to maintain a uniform plant population. Need-based plant protection measures were taken up to raise a healthy crop. Plants were uprooted at harvest. Threshing was done with wooden sticks and seeds winnowed to remove any impurities. Five randomly selected plants from each replication were used for recording of yield attributes. Path coefficients for all possible pairs for seed yield were also computed.

\section{Results and Discussion}

In plant breeding, it is very difficult to have complete knowledge of all component traits of yield. The residual effect permits precise explanation about the pattern of interaction of other possible components of yield. In other words, residual effect measures the role of 
possible independent variables which were not included in the study of dependent variable.

\section{Plant height (cm)}

Plant height recorded negligible and low negative direct effects (-0.047 and -0.172 , respectively) at both genotypic and phenotypic levels on grain yield per plant, while the trait had a positive and significant correlation coefficient $(0.260,0.274)$ with grain yield per plant both at genotypic and phenotypic levels. Plant height exerted moderate and low positive indirect effects via fresh (0.201) and dry weights (0.147) of whole plant, and low negative indirect effects via days taken to maturity $(-0.167)$.

It is inferred by these results that the negative indirect effects were not completely neutralized by the traits contributing towards positive indirect effect and hence the net negative and low range direct effect was shown by plant height on grain yield per plant in coriander. Similar results were obtained by Meena et al., (2014) in coriander and Bandela et al., (2014) for positive direct effect of plant height on seed yield per plant. Banerjee and Kole (2004) also reported negative indirect effects exerted by plant height on pod yield per plant via number of seed per pod in fenugreek.

\section{Number of primary branches per plant}

The trait had negligible negative direct effect $(-0.072,-0.028)$ on dependent variable both at genotypic and phenotypic level. The trait had moderate positive indirect effects through fresh weight (0.246), dry weight (0.145), and moderate negative indirect effects through harvest index (-0.170) and days taken to maturity (-0.135). Negative indirect effect of number of primary branches per plant on grain yield per plant was also reported by Maurya et al., (2015) in fenugreek.

\section{Number of secondary branches}

At genotypic and phenotypic levels, the character exhibited moderate negative direct effect $(-0.224,0.261$ respectively) on grain yield per plant. The trait showed low positive indirect effect through fresh weight (0.107), and had low negative indirect effects through oil content (-0.121). The negative indirect effect could not completely antagonize the positive indirect effect and thus there was a net positive direct effect of this trait on grain yield. Such positive direct effect of number of secondary branches per plant on grain yield per plant was also reported by Anubha et al., (2013) in fenugreek. Similar results were obtained by Mourya et al., (2015) in fenugreek for grain yield per plant for exertion of direct effect on grain yield per plant.

\section{Number of leaves}

This trait showed low positive direct effect on grain yield per plant at genotypic level (0.139) and low negative direct effect at phenotypic level (-0.102). The trait had negligible positive indirect effect on grain yield per plant through days taken to $50 \%$ flowering (0.064), number of secondary branches (0.038), oil content (0.030), and moderate negative indirect effect through days taken to maturity $(-0.215)$.

\section{Leaf area $\left(\mathrm{cm}^{2}\right)$}

Leaf area recorded low and negligible positive direct effect $(0.141,0.022$ respectively) at both genotypic and phenotypic levels. The trait showed low positive indirect effect on grain yield per plant through fresh weight (0.104), while the character had low negative indirect effect though harvest index (-0.145).

\section{Fresh weight of whole plant (g)}

This trait exhibited high positive direct effect on grain yield per plant at genotypic (0.315) 
and phenotypic (0.374). The trait showed moderate positive indirect effect on dependent variable through dry weight $(0.201)$, while the character had low negative indirect effects through harvest index $(-0.135)$ and days taken to maturity (-0.130).

\section{Dry weight of whole plant (g)}

This trait showed moderate and negligible direct effect at genotypic level (0.234) and (0.070) at phenotypic level on grain yield per plant. It exerted moderate positive indirect effect via fresh weight (0.270); and low negative indirect effect via days taken to maturity (-0.173). Similar results were obtained by Bandela et al., (2014) for positive direct effect of dry weight of whole on seed yield per plant in coriander.

\section{Days taken to $50 \%$ flowering}

Negligible and moderate negative direct effect on grain yield per plant was recorded for this trait at genotypic level (-0.094) and (-0.226) at phenotypic level. It exerted high positive indirect effect via days taken to maturity (0.379); and low negative indirect effects via harvest index (-0.189) and dry weight (0.112). Similar results were obtained by Mourya et al., (2015) in fenugreek for grain yield per plant for exertion of direct effects on grain yield per plant.

\section{Number of umbels per plant}

The trait exhibited low positive direct effect on dependent variable at genotypic (0.158) and phenotypic (0.197) levels respectively. It exerted low positive indirect effects via dry weight (0.136) and fresh weight (0.124), and low negative indirect effect via harvest index (-0.116). Similar results were obtained by Mourya et al., (2015) in fenugreek for grain yield per plant for exertion of direct effects on grain yield per plant.

\section{Number of umbellets per umbel}

Number of umbellets per umbel recorded low posative direct effect at genotypic level (0.175) and (0.182) at phenotypic levels on dependent variable respectively. It showed negligible positive indirect effects through fresh weight (0.062), dry weight (0.044) and herbage yield (0.042), and negligible negative indirect effect through days taken to maturity $(-0.090)$ and oil content (-0.072).

\section{Umbel diameter (cm)}

The character showed low positive direct effect on grain yield per plant at genotypic level (0.167) and phenotypic level (0.189). It exerted negligible positive indirect effects via harvest index (0.086) and number of umbellets per umbel (0.074) and low negative indirect effect through days taken to maturity $(-0.119)$.

\section{Number of schizocarps per umbel}

Negligible and low positive direct effect on grain yield per plant was recorded for this trait at genotypic level (0.012) and (0.119) at phenotypic level. It had low positive indirect effect through fresh weight (0.135); and low negative indirect effect through harvest index $(-0.198)$.

\section{Number of schizocarps per plant}

The trait recorded low positive direct effect on grain yield per plant at both genotypic and phenotypic levels $(0.195,0.144)$ respectively. However it exerted a low positive indirect effect via fresh weight (0.101); and low negative indirect effect via days taken to maturity $(-0.114)$.

Similar results were obtained by Patahk et al., (2014) in fenugreek for grain yield per plant for exertion of direct effects on grain yield per plant. 
Table.1 Genotypic path coefficients among different characters of coriander genotypes

\begin{tabular}{|c|c|c|c|c|c|c|c|c|c|c|c|c|c|c|c|c|c|c|}
\hline & PH60 & NPB60 & NSB60 & NL60 & LA60 & FW60 & DW60 & DT50F & NUPP & NULPU & UD & NSPU & NSPP & DTM & HY & HI & 1000SW & $\mathrm{OC}$ \\
\hline PH60 & -0.047 & -0.025 & -0.017 & 0.011 & -0.009 & -0.030 & -0.029 & 0.017 & -0.027 & -0.012 & 0.003 & -0.016 & -0.008 & 0.016 & -0.024 & 0.004 & -0.015 & -0.010 \\
\hline NPB60 & -0.038 & -0.072 & -0.023 & -0.008 & -0.031 & -0.056 & -0.044 & 0.019 & -0.029 & -0.016 & -0.002 & -0.033 & -0.020 & 0.020 & -0.029 & 0.012 & -0.027 & -0.014 \\
\hline NSB60 & -0.083 & -0.073 & -0.224 & 0.038 & -0.090 & -0.076 & -0.075 & 0.033 & -0.073 & -0.005 & -0.008 & -0.050 & -0.026 & 0.030 & -0.085 & -0.021 & 0.015 & 0.063 \\
\hline NL60 & -0.033 & 0.015 & -0.024 & 0.139 & -0.015 & -0.018 & -0.014 & -0.096 & -0.019 & 0.003 & 0.016 & 0.021 & 0.019 & -0.060 & 0.009 & -0.024 & -0.061 & 0.010 \\
\hline LA60 & 0.027 & 0.062 & 0.056 & -0.015 & 0.141 & 0.046 & 0.051 & -0.001 & 0.038 & 0.005 & -0.034 & 0.015 & -0.013 & -0.017 & 0.052 & -0.020 & -0.003 & -0.007 \\
\hline FW60 & 0.201 & 0.246 & 0.107 & -0.041 & 0.104 & 0.315 & 0.270 & -0.093 & 0.124 & 0.062 & -0.008 & 0.135 & 0.101 & -0.082 & 0.229 & -0.043 & 0.203 & 0.071 \\
\hline DW60 & 0.147 & 0.145 & 0.079 & -0.023 & 0.084 & 0.201 & 0.234 & -0.112 & 0.136 & 0.044 & 0.002 & 0.096 & 0.039 & -0.081 & 0.181 & 0.001 & 0.051 & -0.053 \\
\hline DT50F & 0.034 & 0.025 & 0.014 & 0.064 & 0.001 & 0.028 & 0.045 & -0.094 & 0.016 & 0.010 & -0.016 & 0.012 & 0.008 & -0.072 & 0.049 & 0.018 & -0.008 & 0.003 \\
\hline NUPP & 0.090 & 0.065 & 0.052 & -0.022 & 0.043 & 0.062 & 0.092 & -0.026 & 0.158 & -0.022 & -0.033 & 0.051 & -0.014 & 0.009 & 0.044 & -0.018 & 0.040 & 0.003 \\
\hline NULPU & 0.044 & 0.040 & 0.004 & 0.003 & 0.006 & 0.035 & 0.033 & -0.019 & -0.025 & 0.175 & 0.074 & 0.032 & 0.015 & -0.032 & 0.071 & 0.006 & 0.011 & -0.030 \\
\hline UD & -0.011 & 0.005 & 0.006 & 0.019 & -0.040 & -0.004 & 0.001 & 0.028 & -0.035 & 0.070 & 0.167 & -0.005 & 0.023 & -0.040 & -0.032 & 0.014 & 0.005 & -0.012 \\
\hline NSPU & 0.004 & 0.005 & 0.003 & 0.002 & 0.001 & 0.005 & 0.005 & -0.002 & 0.004 & 0.002 & 0.000 & 0.012 & 0.004 & -0.001 & 0.003 & -0.002 & 0.005 & 0.001 \\
\hline NSPP & 0.032 & 0.054 & 0.023 & 0.027 & -0.018 & 0.062 & 0.032 & -0.016 & -0.017 & 0.017 & 0.026 & 0.060 & 0.195 & -0.045 & 0.042 & -0.005 & 0.056 & -0.017 \\
\hline DTM & -0.167 & -0.135 & -0.066 & -0.215 & -0.060 & -0.130 & -0.173 & 0.379 & 0.028 & -0.090 & -0.119 & -0.029 & -0.114 & 0.496 & -0.213 & -0.091 & 0.069 & -0.084 \\
\hline HY & 0.054 & 0.042 & 0.039 & 0.006 & 0.038 & 0.075 & 0.080 & -0.054 & 0.029 & 0.042 & -0.020 & 0.027 & 0.022 & -0.045 & 0.104 & 0.026 & 0.005 & -0.053 \\
\hline HI & -0.094 & -0.170 & 0.093 & -0.176 & -0.145 & -0.135 & 0.006 & -0.189 & -0.116 & 0.033 & 0.086 & -0.198 & -0.027 & -0.183 & 0.255 & 0.922 & -0.009 & -0.494 \\
\hline $1000 \mathrm{SW}$ & 0.007 & 0.008 & -0.001 & -0.009 & 0.000 & 0.014 & 0.005 & 0.002 & 0.005 & 0.001 & 0.001 & 0.009 & 0.006 & 0.003 & 0.001 & 0.000 & 0.021 & 0.001 \\
\hline $\mathrm{OC}$ & 0.093 & 0.081 & -0.121 & 0.030 & -0.022 & 0.096 & -0.098 & -0.014 & 0.009 & -0.072 & -0.031 & 0.023 & -0.037 & -0.073 & -0.218 & -0.211 & 0.021 & 0.429 \\
\hline Residual & alue & & 0.11422 & & & & & & & & & & & & & & & \\
\hline PH60 & \multicolumn{4}{|c|}{ Plant height at $60 \mathrm{DAS}$} & & DT50F & \multicolumn{4}{|c|}{ Days taken to $50 \%$ flowering } & & DTM & \multicolumn{4}{|c|}{ Days taken to maturity } & & \\
\hline NPB60 & \multicolumn{4}{|c|}{ Number of primary branches at 60DAS } & & NUPP & \multicolumn{4}{|c|}{ Number of umbels per plant } & & HY & \multicolumn{4}{|c|}{ Herbage yield } & & \\
\hline NSB60 & \multicolumn{4}{|c|}{ Number of secondary branches at 60DAS } & & NULPU & \multicolumn{4}{|c|}{ Number of umbllets per umbel } & & HI & \multicolumn{4}{|c|}{ Harvest index } & & \\
\hline NL60 & \multicolumn{4}{|c|}{ Number of leaves at 60DAS } & & UD & \multicolumn{4}{|c|}{ Umbel diameter } & & 1000SW & \multicolumn{4}{|c|}{1000 seed weight } & & \\
\hline LA60 & \multicolumn{4}{|c|}{ Leaf area at 60DAS } & & NSPU & \multicolumn{4}{|c|}{ Number of schizocarps per umbel } & & $\mathrm{OC}$ & \multicolumn{4}{|c|}{ Oil content } & & \\
\hline FW60 & \multicolumn{4}{|c|}{ Fresh weight at 60DAS } & & NSPP & \multicolumn{4}{|c|}{ Number of schizocarps per plant } & & GYPP & \multicolumn{4}{|c|}{ Grain yield per plant } & & \\
\hline DW60 & \multicolumn{4}{|c|}{ Dry weight at 60DAS } & & & & & & & & & & & & & & \\
\hline
\end{tabular}


Table.2 Phenotypic path coefficients among different characters of coriander genotypes

\begin{tabular}{|c|c|c|c|c|c|c|c|c|c|c|c|c|c|c|c|c|c|c|}
\hline & PH60 & NPB60 & NSB60 & NL60 & LA60 & FW60 & DW60 & DT50F & NUPP & NULPU & UD & NSPU & NSPP & DTM & HY & HI & 1000SW & OC \\
\hline PH60 & -0.172 & -0.080 & -0.065 & 0.009 & -0.041 & -0.107 & -0.101 & 0.028 & -0.098 & -0.053 & -0.004 & -0.062 & -0.029 & 0.039 & -0.072 & 0.011 & -0.058 & -0.038 \\
\hline NPB60 & -0.013 & -0.028 & -0.014 & -0.004 & -0.011 & -0.019 & -0.016 & 0.006 & -0.009 & -0.005 & -0.001 & -0.010 & -0.008 & 0.004 & -0.015 & 0.003 & -0.016 & -0.011 \\
\hline NSB60 & -0.099 & -0.129 & -0.261 & -0.019 & -0.082 & -0.071 & -0.100 & -0.008 & -0.071 & -0.046 & -0.006 & -0.059 & -0.040 & 0.000 & -0.122 & -0.032 & -0.111 & -0.018 \\
\hline NL60 & 0.006 & -0.013 & -0.008 & -0.102 & -0.002 & 0.007 & -0.002 & 0.019 & 0.005 & -0.025 & -0.013 & -0.025 & -0.012 & 0.013 & -0.008 & 0.008 & 0.004 & -0.010 \\
\hline LA60 & 0.005 & 0.008 & 0.007 & 0.000 & 0.022 & 0.007 & 0.007 & 0.002 & 0.006 & 0.002 & -0.001 & 0.003 & -0.001 & -0.001 & 0.007 & -0.003 & 0.001 & 0.000 \\
\hline FW60 & 0.232 & 0.251 & 0.101 & -0.026 & 0.121 & 0.374 & 0.290 & -0.081 & 0.145 & 0.070 & -0.003 & 0.155 & 0.117 & -0.080 & 0.220 & -0.047 & 0.179 & 0.080 \\
\hline DW60 & 0.041 & 0.040 & 0.027 & 0.001 & 0.021 & 0.054 & 0.070 & -0.024 & 0.036 & 0.012 & 0.000 & 0.028 & 0.011 & -0.016 & 0.049 & 0.002 & 0.021 & -0.007 \\
\hline DT50F & 0.037 & 0.048 & -0.007 & 0.041 & -0.017 & 0.049 & 0.077 & -0.226 & 0.015 & -0.040 & -0.036 & -0.005 & 0.017 & -0.168 & 0.093 & 0.022 & -0.041 & 0.008 \\
\hline NUPP & 0.112 & 0.065 & 0.054 & -0.010 & 0.052 & 0.077 & 0.102 & -0.013 & 0.197 & -0.011 & -0.031 & 0.065 & -0.018 & 0.016 & 0.038 & -0.019 & 0.041 & 0.003 \\
\hline NULPU & 0.057 & 0.032 & 0.032 & 0.045 & 0.014 & 0.034 & 0.032 & 0.032 & -0.010 & 0.182 & 0.060 & 0.049 & 0.013 & -0.005 & 0.046 & 0.015 & 0.038 & -0.021 \\
\hline UD & 0.004 & 0.005 & 0.004 & 0.024 & -0.005 & -0.001 & -0.001 & 0.030 & -0.030 & 0.062 & 0.189 & -0.001 & 0.030 & -0.029 & -0.024 & 0.013 & 0.007 & -0.008 \\
\hline NSPU & 0.043 & 0.044 & 0.027 & 0.030 & 0.015 & 0.050 & 0.047 & 0.002 & 0.040 & 0.032 & -0.001 & 0.119 & 0.034 & 0.002 & 0.028 & -0.019 & 0.045 & 0.005 \\
\hline NSPP & 0.024 & 0.043 & 0.022 & 0.017 & -0.009 & 0.045 & 0.023 & -0.011 & -0.013 & 0.010 & 0.023 & 0.041 & 0.144 & -0.029 & 0.035 & -0.003 & 0.040 & -0.002 \\
\hline DTM & -0.086 & -0.059 & 0.000 & -0.048 & -0.009 & -0.081 & -0.088 & 0.282 & 0.031 & -0.010 & -0.057 & 0.006 & -0.076 & 0.379 & -0.100 & -0.049 & 0.086 & -0.034 \\
\hline HY & 0.088 & 0.112 & 0.099 & 0.017 & 0.063 & 0.125 & 0.149 & -0.087 & 0.041 & 0.053 & -0.026 & 0.050 & 0.051 & -0.056 & 0.211 & 0.046 & 0.067 & -0.031 \\
\hline HI & -0.052 & -0.096 & 0.101 & -0.063 & -0.099 & -0.103 & 0.025 & -0.080 & -0.080 & 0.069 & 0.054 & -0.128 & -0.018 & -0.106 & 0.178 & 0.815 & 0.047 & -0.335 \\
\hline 1000SW & -0.031 & -0.051 & -0.039 & 0.003 & -0.005 & -0.044 & -0.027 & -0.016 & -0.019 & -0.019 & -0.003 & -0.034 & -0.025 & -0.021 & -0.029 & -0.005 & -0.091 & -0.029 \\
\hline $\mathrm{OC}$ & 0.077 & 0.135 & 0.023 & 0.035 & -0.003 & 0.074 & -0.033 & -0.013 & 0.005 & -0.040 & -0.015 & 0.015 & -0.005 & -0.031 & -0.051 & -0.143 & 0.112 & 0.348 \\
\hline \multicolumn{3}{|c|}{ Residual value } & 0.176 & & & & & & & & & & & & & & & \\
\hline PH60 & \multicolumn{4}{|c|}{ Plant height at 60DAS } & & DT50F & \multicolumn{4}{|c|}{ Days taken to $50 \%$ flowering } & & DTM & \multicolumn{4}{|c|}{ Days taken to maturity } & & \\
\hline NPB60 & \multicolumn{4}{|c|}{ Number of primary branches at 60DAS } & & NUPP & \multicolumn{4}{|c|}{ Number of umbels per plant } & & HY & \multicolumn{4}{|c|}{ Herbage yield } & & \\
\hline NSB60 & \multicolumn{4}{|c|}{ Number of secondary branches at 60DAS } & & NULPU & \multicolumn{4}{|c|}{ Number of umbllets per umbel } & & HI & \multicolumn{4}{|c|}{ Harvest index } & & \\
\hline NL60 & \multicolumn{4}{|c|}{ Number of leaves at 60DAS } & & UD & \multicolumn{4}{|c|}{ Umbel diameter } & & $1000 \mathrm{SW}$ & \multicolumn{4}{|c|}{1000 seed weight } & & \\
\hline LA60 & \multicolumn{4}{|c|}{ Leaf area at 60DAS } & & NSPU & \multicolumn{4}{|c|}{ Number of schizocarps per umbel } & & $\mathrm{OC}$ & \multicolumn{4}{|c|}{ Oil content } & & \\
\hline FW60 & \multirow{2}{*}{\multicolumn{4}{|c|}{$\begin{array}{l}\text { Fresh weight at 60DAS } \\
\text { Dry weight at 60DAS }\end{array}$}} & & NSPP & \multicolumn{4}{|c|}{ Number of schizocarps per plant } & & GYPP & \multicolumn{4}{|c|}{ Grain yield per plant } & & \\
\hline DW60 & & & & & & & & & & & & & & & & & & \\
\hline
\end{tabular}




\section{Days taken to maturity}

The trait exhibited high positive direct effect on dependent variable at genotypic level (0.496) and phenotypic level (0.379). It exerted negligible positive indirect effects via number of secondary branches (0.030), number of primary branches $(0.020)$ and plant height (0.016); and low negative indirect effect via harvest index $(-0.183)$.

\section{Herbage yield (g)}

Low and High moderate direct effect on grain yield per plant was recorded for this character (0.104) at genotypic level and (0.211) at phenotypic level.

It showed moderate positive indirect effects on dependent variable through harvest index (0.255) and fresh weight (0229); and moderate negative indirect effects via oil content (-0.218) and days taken to maturity ($0.213)$.

\section{Harvest index (\%)}

The trait recorded high positive direct effect on grain yield per plant at genotypic level (0.922) and at phenotypic level (0.815), with significance and positive correlation coefficient of 0.648 at genotypic level.

However the trait exerted negligible positive indirect effects on grain yield per plant via herbage yield (0.026) and umbel diameter (0.014); and moderate negative indirect effect via oil content (-0.218). Similar results were obtained by Bandela et al., (2014) for positive direct effect of dry weight of whole on seed yield per plant in coriander.

\section{Thousand-grain weight (g)}

The trait recorded negligible positive direct effect on grain yield per plant at genotypic level (0.021) and negligible negative direct effect on grain yield per plant at phenotypic level (-0.091). However the trait exerted moderate positive indirect effects on grain yield per plant via fresh weight (0.203); and negligible negative indirect effect via number of leaves (-0.061), number of primary branches (-0.027) and plant height (-0.015). Similar results were obtained by Mourya et al., (2015) in fenugreek for grain yield per plant for exertion of direct effects on grain yield per plant.

\section{Oil content (\%)}

The trait recorded high positive direct effect on grain yield per plant both at genotypic level (0.429) and at phenotypic level (0.348).

However the trait exerted negligible positive indirect effects on grain yield per plant via fresh weight (0.071) and number of secondary branches (0.063); and high negative indirect effect via harvest index (-0.494).

The path analysis indicated that leaf area, fresh weight, dry weight, number of umbels per plant, number of umbellets per umbel, umbel diameter, number of schizocarps per umbel, number of schizocarps per plant, days taken to maturity, herbage yield, harvest index, thousand seed weight and oil content had direct positive effects on fruit yield per plant at phenotypic and genotypic levels (Table 1 and 2).

Based on the results of path analysis, the present study revealed that major emphasis should be laid on selection process with more number of leaves, leaf area, fresh weight, dry weight, number of umbels per plant, number of umbellets per umbel, umbel diameter, number of schizocarps per plant, days taken to maturity, herbage yield, harvest index and oil content and there should be economic balance among these traits to get higher grain yield per plant. 


\section{References}

Anubha, J, Balraj Singh, Solanki K.R, Saxena, N.S. and Kale, K.R. 2013. Genetic variability and character association in fenugreek (Trigonell foenum-graecum L.). International Journal of Seed Spices. 3(2): 22-28.

Bandela, S.B, Sreeramu, B.S, Narsimha, S.B, Umesha, K. and Rajasekhar, R. 2014. Correlation coefficient and path analysis in coriander (Coriandrum sativum L.). International Journal of Applied Biology and Pharmaceutical Technology. 5(4): 60-62.

Banerjee. and Kole, C.P. 2004. Genetic variability, correlation and path analysis in fenugreek (Trigonell foenum-graecum L.). Journal of Spices and Aromatic Crops. 13(1): 44-48.

Datta, S. and Choudhuri, P. 2006. Evaluation of coriander germplasm under Terai zone of West Bengal. Haryana Journal of Horticultural Sciences, 35: 348-349.

Gomez, K., and Gomez, A. 1984. Statistical Procedures for Agricultural Research. Wiley Interscience, New York, USA.

Malik, T.P. and Tehlan, S.K. 2013. Performance of coriander (Coriandrum sativum L.) varieties for growth and seed yield. International Journal of Seed Spices, 3: 8990.

Meena, K.Y, Kale S.V. and Meena P.O. 2014. Correlation coefficient and path analysis in coriander. International Journal of Scientific and Research Publications. 4(6): 2250-3153.

Mengesha, B., and Getinetalemaw, G. 2010. Variability in Ethiopian coriander accessions for agronomic and quality traits. African Crop Science Journal, 18 (2): 43-49.

Moniruzzaman, M., Rahman, M.M., Hossain, M.M., Karim, A.S. and Khaliq, Q.A. 2013. Evaluation of coriander (Coriandrum sativum L.) genotypes for seed yield and yield contributing characters. Bangladesh Journal of Agricultural Research, 38: 189-202.

Morales-Payan, J.P. 2011. Herbs and leaf crops: Cilantro, broadleaf cilantro and vegetable amaranth. pp. 1-28. In: Soils, Plant Growth and Corp Production, Vol. 3 (Ed. W.H. Verheye). Eolss Publishers, Oxford, UK.

Mourya, P.B, Yadav, K.B, Pandey, P.V. and Yadav S.P. 2015. Correlation and path analysis in Fenugreek (Trigonella foenumgraecum L.). Research of Environmental Life Science. 8(4): 569-70.

NHB. 2014. Commodity wise Status. Indian Horticulture Database. National Horticulture Board, New Delhi.

Nilkolay, D. and Boryana, D. 2014. Heritability and correlation coefficient analysis for fruit yield and its components in coriander (Coriandrum sativum L.). Turkish Journal of Agricultural and Natural Sciences. 1: 618-22.

Patahk, R.A, Patil, I.A, Joshi, K.H. and Patil, A.D. 2014. Genetic variability, correlation and coefficient analysis in fenugreek (Trigonell foenum-graecum L.). Trends in Biosciences. 7(4): 234-37.

Sarada, C. and Kalidasu, G. 2009. Elite genotypes of coriander suitable for rain fed cultivation in Andhra Pradesh. Annals of Plant Physiology, 23: 174-176.

Sarada, C. and Kalidasu, G. 2011. Threats in production of coriander (Coriandrum sativum) in Andhra Pradesh. Journal of Spices and Aromatic Crops, 17: 158-162.

Sharma, M.M. and Sharma, R.K. 2004. Coriander. pp. 145-161. In: Hand book of Herbs and Spices. (Ed. K.V. Peter). Woodhead Publishing Limited, Cambridge, England.

Tiwari, R.K. 2014. Crop-wise area, production and productivity of major spice crops in India during 2010-11, 2011-12 and 2012-13. In: Indian Horticulture Database, 2013 (Eds.) N.C.

\section{How to cite this article:}

Nagappa, M.K., N. Emmanuel, M. Lakshminarayana Reddy and Dorajeerao, A.V.D. 2018. Path Coefficient Analysis for Grain and Oil Yield in Coriander. Int.J.Curr.Microbiol.App.Sci. 7(01): 1534-1541. doi: https://doi.org/10.20546/ijcmas.2018.701.186 